\title{
Reversible Formation of Interpenetrating Polymer Network Structure in Organic-Inorganic Polymer Hybrids
}

\author{
Yusuke ImaI, Kensuke NaKa, and Yoshiki CHUjo ${ }^{\dagger}$ \\ Department of Polymer Chemistry, Graduate School of Engineering, \\ Kyoto University, Yoshida, Sakyo-ku, Kyoto 606-8501, Japan
}

(Received June 3, 1998)

\begin{abstract}
A new strategy to prepare organic inorganic polymer hybrids having interpenetrating polymer network (IPN) structure is presented. Polymer hybrids were synthesized from poly(2-methyl-2-oxazoline) having coumarin moieties as reversibly photo-crosslinkable side groups (4). It was found that the concentration of acid catalyst for sol-gel reaction of tetramethoxysilane (TMOS) was essential to obtain transparent and homogeneous polymer hybrids. Reversible photodimerization of coumarin groups took place in the polymer hybrids by the irradiation of light with different wavelengths. The resulting polymer hybrids are IPN of organic gel and silica gel. Solvent-resistant property of the polymer hybrids was improved by formation of the IPN structure.
\end{abstract}

KEY WORDS Organic-Inorganic Polymer Hybrids / Sol-Gel Reaction / Poly(2-methyl-2-oxazoline) / Coumarin / Reversible Photo-Dimerization / Interpenetrating Polymer Network / Solvent-Resistant Property /

Interpenetrating polymer networks, IPNs, are a part of polymer blends where two or more polymers are combined, with at least one of the polymers crosslinked in the presence of the other(s). They are known to have distinct morphology and to exhibit properties different from only a sum of the original polymers or polymer gels. ${ }^{1}$ In a narrow sense, compositions where each and all the polymers form networks are called IPN. On the other hand, polymer blends where one polymer is crosslinked while another remains linear are defined as semiIPN. Composite materials consist of organic polymers and inorganic silica gel can be considered as a kind of IPN materials. ${ }^{2,3}$ In our laboratory, organic-inorganic polymer hybrids having IPN structure have been synthesized by the in situ polymerization method. ${ }^{4,5}$ In this method, the sol-gel reaction of alkoxysilanes and radical copolymerization of organic monomers (and crosslink agent) are conducted simultaneously in situ. They showed high thermal and/or mechanical stability and solventresistant property.

Here we present another method for the preparation of organic-inorganic polymer hybrids with an IPN structure (Scheme 1). Organic polymers having pendant crosslinkable functional groups can form a threedimensional polymer network (gel) by a crosslinking reaction. Furthermore, if the reaction is reversible in response to external stimuli, it becomes possible to build the gel-structure reversibly. Coumarin derivative is known to photo-dimerize upon irradiation by ultraviolet light with wavelengths longer than $310 \mathrm{~nm},{ }^{6,7}$ and the reverse reaction (photo-cleavage) proceeds by the light of shorter wavelengths than $310 \mathrm{~nm} .^{8-10}$ Poly(2-methyl2-oxazoline) with pendant coumarin groups (4) has been prepared and its reversible gel-formation was reported previously. ${ }^{11}$ In the present study, the preparation of organic-inorganic polymer hybrids from polymer $\mathbf{4}$ and the reversible photo-crosslinking reaction in the polymer hybrids are described (Scheme 2). The polymer hybrids form semi-IPN structure and IPN structure reversibly by the irradiation of light with different wavelengths. Changes in spectroscopic, thermal, and solvent-resistant properties were investigated.

\section{EXPERIMENTAL}

\section{Materials}

Tetramethoxysilane (TMOS), methyltrimethoxysilane (MeTMOS), and phenyltrimethoxysilane (PhTMOS) were distilled and stored under nitrogen atmosphere. Methanol was dried and distilled from magnesium methoxide, and stored under nitrogen atmosphere. Aqueous hydrochloric acid of various concentrations and $N, N$-dimethylformamide (DMF) were used as received.

\section{Measurements}

${ }^{1} \mathrm{H}$ NMR spectra were recorded on a $270 \mathrm{MHz}$ JEOLJNM-GX270 NMR spectrometer. Absorption spectra
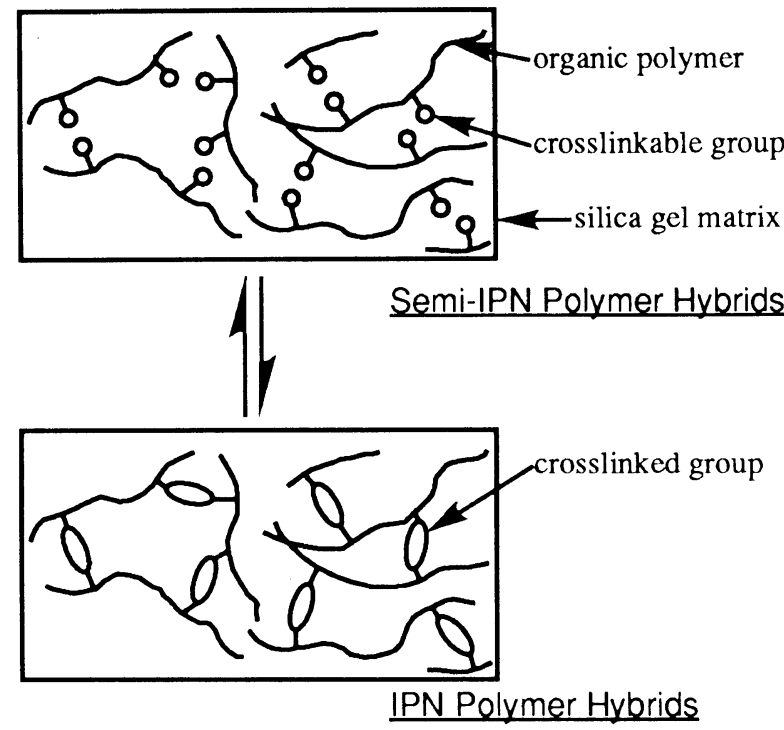

Scheme 1.

${ }^{\dagger}$ To whom correspondence should be addressed. 


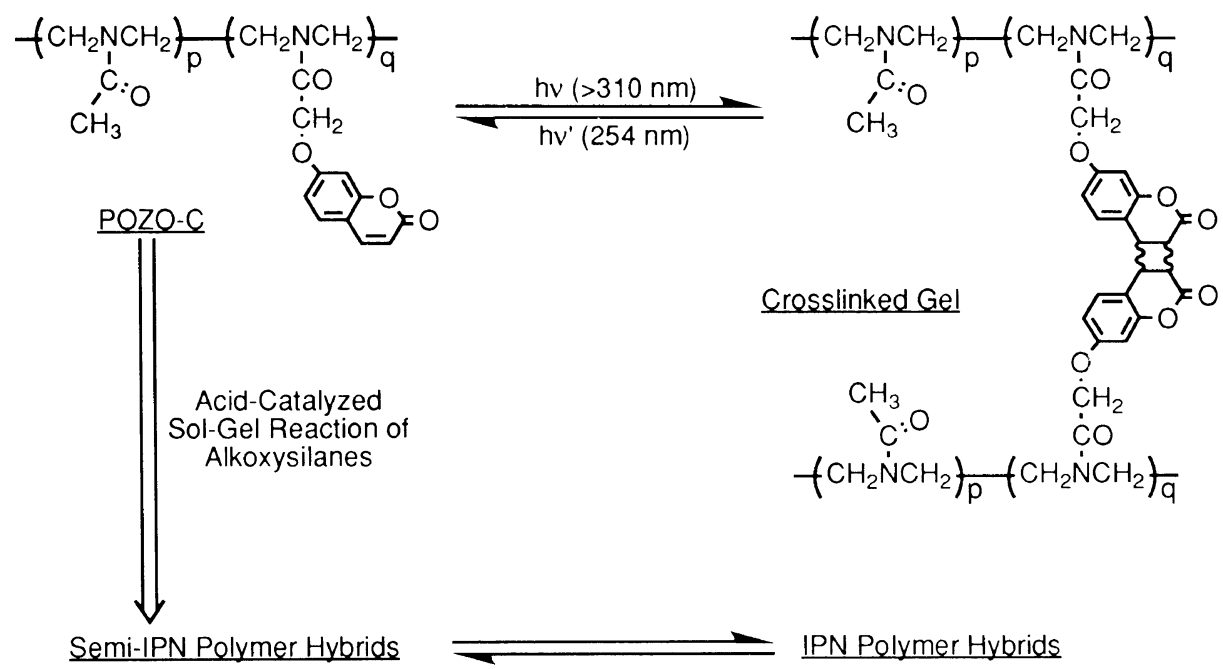

Scheme 2 .

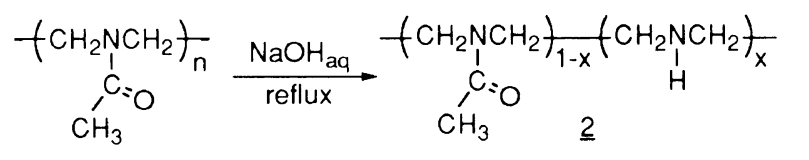

1

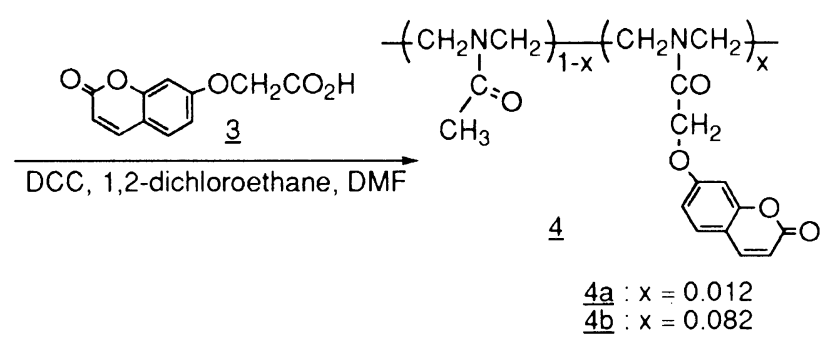

Scheme 3 .

were obtained on a JASCO V-530 spectrophotometer. Differential scanning calorimetry (DSC) thermograms were obtained with a DSC200, Seiko Instruments Inc., with the heating rate of $15^{\circ} \mathrm{Cmin}^{-1}$ under nitrogen atmosphere. Thermogravimetric analysis (TGA) was performed on a Shimadzu DT-30.

\section{Preparation of Polymer Hybrids from Polymer 4}

Polymer 4 was prepared by the condensation reaction of 7-coumariloxyacetic acid and partially hydrolyzed poly(2-methyl-2-oxazoline), as illustrated in Scheme 3. Details of synthetic procedure are described previously. ${ }^{11}$ Number average molecular weight was about 8500 and degree of modification was determined from ${ }^{1} \mathrm{H}$ NMR and absorption spectra. Two polymers with the different degree of modification was prepared (4a and $\mathbf{4 b}$ ). A typical procedure for the preparation of polymer hybrids from polymer $\mathbf{4}$ is as follows. Polymer $\mathbf{4}$ and TMOS were dissolved in methanol and aqueous hydrochloric acid was added. The resulting mixture was placed in a polypropylene mold covered with a paper towel and left in air at room temperature for 1 week and at $60^{\circ} \mathrm{C}$ for another week. After complete removal of the solvent by evaporation, polymer hybrids were obtained as glassy solid.
Photo-Dimerization and Photo-Cleavage Reactions

Polymer hybrid films for photochemical reactions were prepared by casting the reaction mixture on quartz substrates $(10 \mathrm{~mm} \times 45 \mathrm{~mm})$. Ethylene glycol was added to the solution as a drying control chemical additive (DCCA) to prevent cracking. ${ }^{12}$ The cast films were placed at room temperature for several days, heated at $60^{\circ} \mathrm{C}$ for $12 \mathrm{~h}$, and then heated at $80^{\circ} \mathrm{C}$ under reduced pressure for $1 \mathrm{~h}$. No cracks were observed on the films. Film thickness was $c a .100 \mu \mathrm{m}$. Films of polymer $\mathbf{4 a}$ and $\mathbf{4 b}$ were also prepared on quartz substrates by casting the methanol solution of polymer. After removing the solvent by evaporation, the films were dried in vacuo at $70^{\circ} \mathrm{C}$ for $12 \mathrm{~h}$.

Photo-dimerization of coumarin groups was induced by the irradiation of light longer than $310 \mathrm{~nm}$ by a $450 \mathrm{~W}$ high-pressure mercury lamp through a pylex cooler. Photo-cleavage reaction was carried out by the irradiation at $254 \mathrm{~nm}$ by a $10 \mathrm{~W}$ low-pressure mercury lamp. Both reactions were performed at room temperature, and followed by the absorption spectra.

\section{Solvent-Resistant Property of Polymer Hybrids}

Polymer hybrids were ground in a mortar and the resulting powders were extracted with methanol by using Soxhlet apparatus for 1 week, or stirred in DMF for 1 week. The quantity of the organic component remaining in the polymer hybrids after extraction was evaluated from the elemental analysis of nitrogen and the thermogravimetric analysis in air.

\section{RESULTS AND DISCUSSION}

\section{Synthesis of Polymer Hybrids from Polymer 4}

Table I summarizes the results of the preparation of the polymer hybrids from polymer 4 . Methanol was used as a solvent and three kinds of alkoxysilanes were employed. The effect of acid concentration was also examined. It was found that the higher acid concentration gave the higher homogeneity of the polymer hybrids when TMOS was used as the alkoxysilane (runs 1-4). And with 1 molar hydrochloric acid, transparent and homogeneous polymer hybrids could be obtained in a wide 
Table I. Organic-inorganic polymer hybrids from polymer $\mathbf{4}^{\mathrm{a}}$

\begin{tabular}{rccccl}
\hline Run & Polymer $^{\mathbf{b}}$ & Alkoxysilane & Ratio $^{\mathbf{c}}$ & $\mathrm{HCl}_{\mathbf{a q}}{ }^{\mathbf{d}}$ & Appearance \\
\hline 1 & $\mathbf{4 a}$ & TMOS & $1 / 20$ & $0.001 \mathrm{M}$ & Turbid \\
2 & $\mathbf{4 a}$ & TMOS & $1 / 20$ & $0.01 \mathrm{M}$ & Translucent \\
3 & $\mathbf{4 a}$ & TMOS & $1 / 20$ & $0.1 \mathrm{M}$ & Translucent \\
4 & $\mathbf{4 a}$ & TMOS & $1 / 20$ & $1 \mathrm{M}$ & Transparent \\
5 & $\mathbf{4 a}$ & TMOS & $1 / 10$ & $1 \mathrm{M}$ & Transparent \\
6 & $\mathbf{4 a}$ & TMOS & $1 / 4$ & $1 \mathrm{M}$ & Transparent \\
7 & $\mathbf{4 a}$ & TMOS & $1 / 2$ & $1 \mathrm{M}$ & Transparent \\
8 & $\mathbf{4 a}$ & TMOS & $1 / 1$ & $1 \mathrm{M}$ & Transparent \\
\hline 9 & $\mathbf{4 b}$ & TMOS & $1 / 20$ & $1 \mathrm{M}$ & Transparent \\
10 & $\mathbf{4 b}$ & TMOS & $1 / 10$ & $1 \mathrm{M}$ & Transparent \\
11 & $\mathbf{4 b}$ & TMOS & $1 / 4$ & $1 \mathrm{M}$ & Phase separated \\
12 & $\mathbf{4 b}$ & TMOS & $1 / 2$ & $1 \mathrm{M}$ & Phase separated \\
\hline 13 & $\mathbf{4 a}$ & MeTMOS & $1 / 20$ & $0.001 \mathrm{M}$ & Turbid \\
14 & $\mathbf{4 a}$ & MeTMOS & $1 / 20$ & $0.01 \mathrm{M}$ & Translucent \\
15 & $\mathbf{4 a}$ & MeTMOS & $1 / 20$ & $0.1 \mathrm{M}$ & Turbid \\
16 & $\mathbf{4 a}$ & MeTMOS & $1 / 20$ & $1 \mathrm{M}$ & Translucent \\
\hline 17 & $\mathbf{4 a}$ & PhTMOS & $1 / 20$ & $0.01 \mathrm{M}$ & Phase separated \\
18 & $\mathbf{4 a}$ & PhTMOS & $1 / 20$ & $0.1 \mathrm{M}$ & Translucent \\
19 & $\mathbf{4 a}$ & PhTMOS & $1 / 20$ & $1 \mathrm{M}$ & Translucent \\
& & & & \\
\hline
\end{tabular}

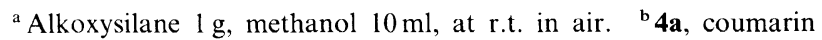
content $1.2 \mathrm{~mol} \% ; \mathbf{4 b}$, coumarin content $8.2 \mathrm{~mol} \%$. ${ }^{\mathrm{c}}$ Feed ratio of polymer to alkoxysilane $(\mathrm{w} / \mathrm{w})$. d 4 eq to TMOS; 3 eq to MeTMOS and PhTMOS.

range of the feed ratios of $\mathbf{4 a}$ to TMOS (runs $4-8$ ). On the contrary, it was difficult to obtain homogeneous polymer hybrids with MeTMOS and PhTMOS (runs 13-19).

These results are somewhat different from the previous study with poly(2-methyl-2-oxazoline) (1), where the transparent material could be obtained easily not only with TMOS but with MeTMOS and PhTMOS. ${ }^{13}$ Only $1.2 \mathrm{~mol} \%$ modification of 1 with coumarin group largely affected the homogeneity of the polymer hybrids. This may be attributed to the hydrophobicity of the coumarin moieties. Polymer $\mathbf{4}$ dissolves well in methanol in the first stage of the reaction. It is considered that the low homogeneity of the resulting polymer hybrids is the result of the aggregation of coumarin groups during the sol-gel reaction and the evaporation of methanol due to their hydrophobicity. The sol-gel reaction of alkoxysilanes, that is, the hydrolysis and the condensation reactions, is accelerated with higher acid concentration. Consequently, the gelation took place faster. This acceleration effect allows the reaction mixture to be frozen before macroscopic phase separation, and the homogeneous polymer hybrids could be obtained only with 1 molar hydrochloric acid. The low homogeneity with MeTMOS and PhTMOS can be explained by slower gelation with tri-functional MeTMOS and PhTMOS than tetra-functional TMOS. It seems that the tendency of aggregation of coumarin also causes high yield of the photodimerization of coumarin in the polymer hybrids. This will be discussed in later section.

Preparation of polymer hybrids from higher coumarincontent polymer $\mathbf{4 b}(8.2 \mathrm{~mol} \%)$ was also examined (runs 9-12). Transparent polymer hybrids could be obtained only with lower polymer ratio, and higher polymer ratio brought about the phase separation. The effect of ag992

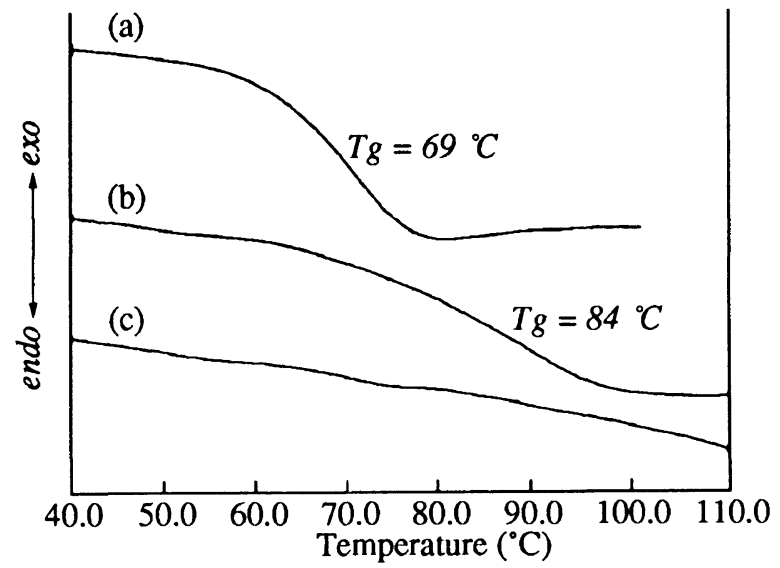

Figure 1. DSC thermograms of (a) polymer $\mathbf{4 b}$ before the crosslink, (b) polymer $\mathbf{4 b}$ after the crosslink, and (c) polymer $\mathbf{4 b} /$ TMOS polymer hybrid (run 10).

gregation of coumarin groups should dominate in these cases.

Usually many cracks appeared in the polymer hybrids from TMOS. They could be avoided by adding ethylene glycol to the reaction mixture as a drying control chemical additive, ${ }^{12}$ and crackless films were prepared on quartz substrates. In the following section these films were used to investigate the reversible photo-dimerization reactions of the coumarin groups in the polymer hybrids spectroscopically.

Glass transition behavior of polymer $\mathbf{4}$ and the polymer hybrids were studied using DSC. Figure 1 shows representatively the DSC thermograms of polymer $\mathbf{4 b}$ and polymer hybrid from $\mathbf{4 b}$ (run 10). Glass transition temperature $\left(T_{\mathrm{g}}\right)$ of $\mathbf{4 b}$ was observed at $69^{\circ} \mathrm{C}$ (Figure 1a). On the other hand, as shown in Figure 1c, glass transition was not distinguished in the DSC thermogram of the polymer hybrid. This observation indicates that polymer 4 is dispersed at the molecular level in silica gel network.

\section{Photo-Dimerization of Coumarin Groups in the Polymer Hybrids}

The photo-dimerization of the coumarin groups was induced by the light of longer wavelengths than $310 \mathrm{~nm}$ by a high-pressure mercury lamp. The reaction was carried out on all the transparent polymer hybrid films (runs $4-10$ ) as well as the films of polymer $\mathbf{4 a}$ and $\mathbf{4 b}$. Almost quantitative reaction was confirmed by absorption spectra in all the samples. Figure 2 shows the typical change of the absorption spectra of polymer hybrid film upon the irradiation. Decline of the absorption at $322 \mathrm{~nm}$ indicates the progress of photo-dimerization and the formation of IPN structure in the polymer hybrids. The reaction took place almost quantitatively even with the lowest coumarin content sample (run 4), suggesting that the coumarin groups exist adjacent to each other in the polymer hybrids. This corroborates the aggregation of the coumarin groups in the polymer hybrids, which was presumed from the results of the preparation of the polymer hybrids. No change was found in the appearance of the polymer hybrid films during the irradiation. 


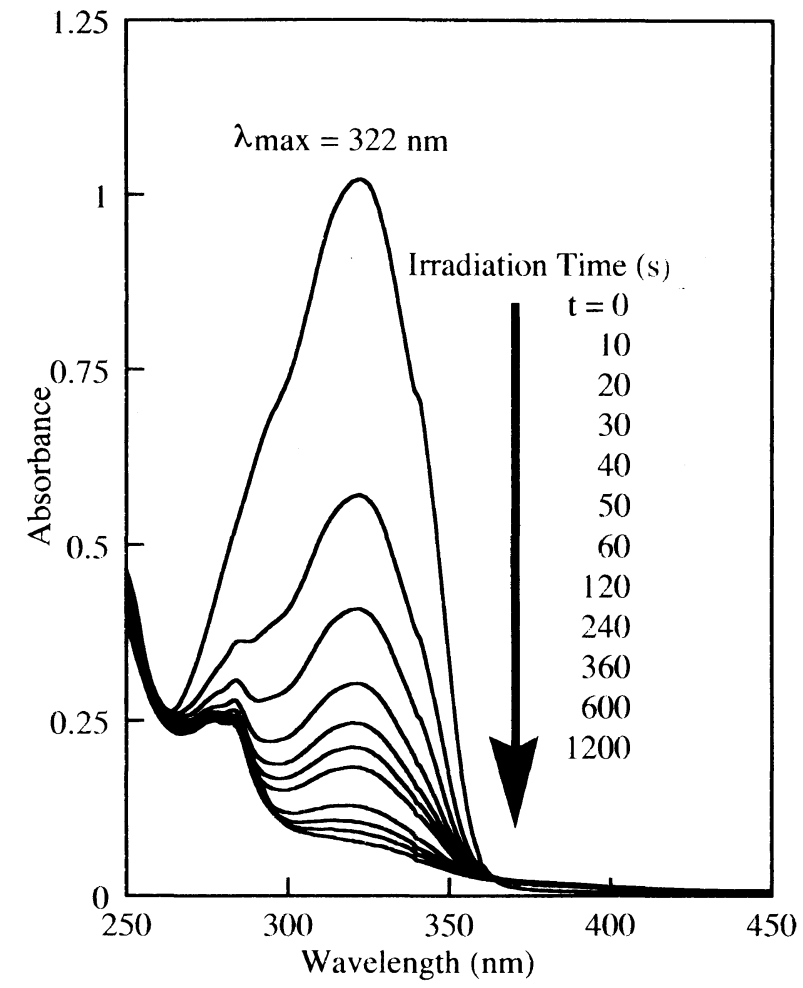

Figure 2. The change of absorption spectra of the polymer hybrid (run 6) upon irradiation of light longer than $310 \mathrm{~nm}$ by a $450 \mathrm{~W}$ high-pressure mercury lamp.

\section{Thermal and Solvent-Resistant Properties of Polymer Hybrids \\ $T_{\mathrm{g}}$ of polymer $4 \mathrm{~b}$ rose from $69^{\circ} \mathrm{C}$ to $84^{\circ} \mathrm{C}$ and the} transition became broader after the photo-dimerization (Figures $1 \mathrm{a}$ and $1 \mathrm{~b}$ ). In other words, the photo-dimerization, namely, the crosslink of the polymer main chain, affected the thermal property of the polymer. On the other hand, as polymer $\mathbf{4}$ is dispersed completely in the polymer hybrids, it is difficult to expect any change of thermal property of the polymer hybrids by the crosslink. Glass transition was hardly observed by DSC on all the transparent polymer hybrids (runs 4-10), as representatively shown in Figure 1c (run 10). TGA study was also carried out, but no remarkable change of thermal stability was found. The crosslink of polymer 4 seems to have little effect on the thermal properties of the polymer hybrids.

Solvent-resistant property is another interest, as the IPN polymer hybrids have been found to have excellent solvent-resistant property compared with the traditional ones. ${ }^{14}$ The solvent-resistant properties of the polymer hybrids before and after the crosslink were compared with each other from remained polymer contents after extraction with methanol or DMF. Figure 3 shows the results of the extraction experiment on the polymer hybrids from low-coumarin-content polymer 4a. Solventresistant property seems to be improved by the crosslink in all the samples. Most of the organic polymer was, however, extracted with methanol even after the crosslink. It is considered that the crosslink density is too small to prevent the extraction. This is supported by the fact that the polymer $\mathbf{4 a}$ dissolves in methanol even after the complete crosslink.

On the contrary, in the case of the polymer hybrids

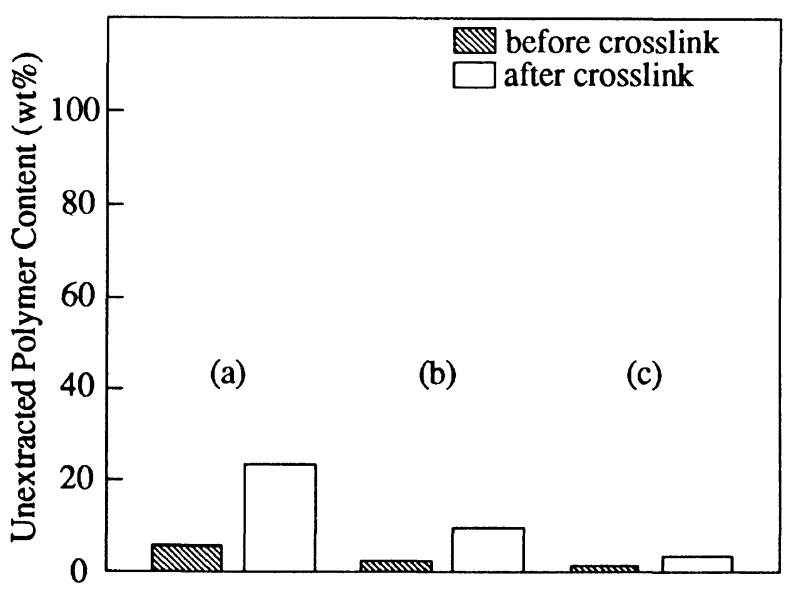

Figure 3. Solvent-resistant property of the polymer hybrids from polymer 4a. Ground samples were extracted with methanol by using Soxhlet apparatus for 1 week. (a) run $5(\mathbf{4 a} / \mathrm{TMOS}=1 / 10)$; (b) run 6 $(1 / 4)$; (c) run $7(1 / 2)$

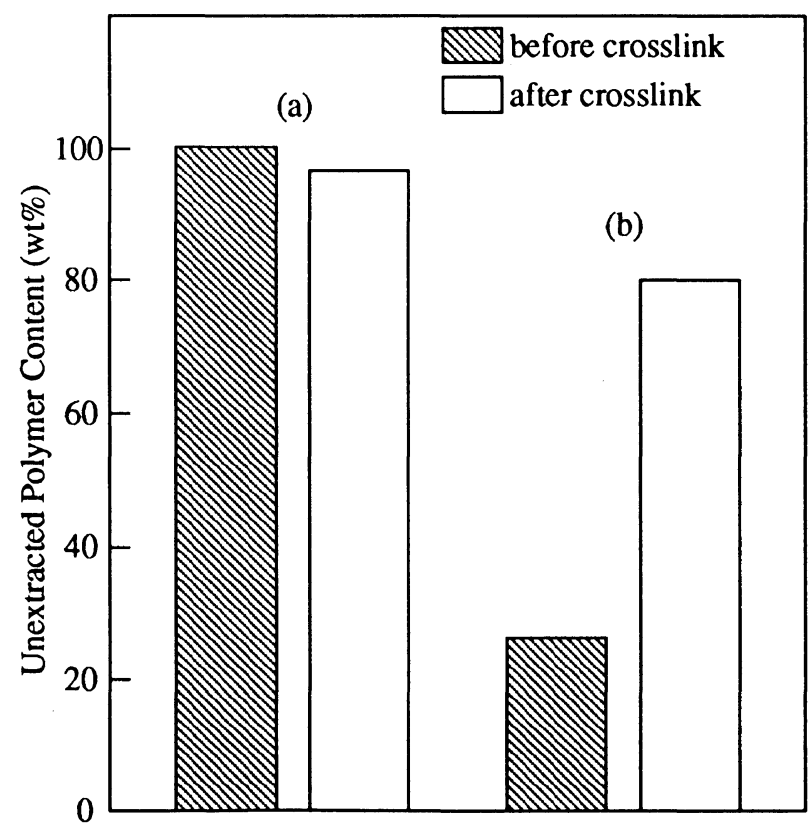

Figure 4. Solvent-resistant property of the polymer hybrids from polymer $\mathbf{4 b}$. Ground sample (run 10) was extracted with (a) methanol and (b) DMF.

from high-coumarin-content polymer $\mathbf{4 b}$, organic component was hardly extracted with methanol even before the crosslink (Figure 4a). Generally, as exemplified in the case of the polymer hybrids from polymer $\mathbf{4 a}$, linear polymer can easily be extracted from polymer hybrids by hydrogen-bond donating solvents like methanol. There are two possibilities to explain this unusual solvent-resistant behavior of uncrosslinked polymer hybrids from $\mathbf{4 b}$. One is that the lactone ring of coumarin group is hydrolyzed in acidic reaction media, and form covalent bond with silica matrix through condensation between silanol groups on silica gel and generated alcohol or carboxylic acid groups. Another possibility is that the main chain of polymer $\mathbf{4 b}$ is physically linked by the aggregates of coumarin groups. As described in the previous sections, the coumarin groups are supposed to aggregate to some extent in the polymer hybrids owing to their hydrophobicity. Polymer $\mathbf{4 b}$ itself dissolves in 


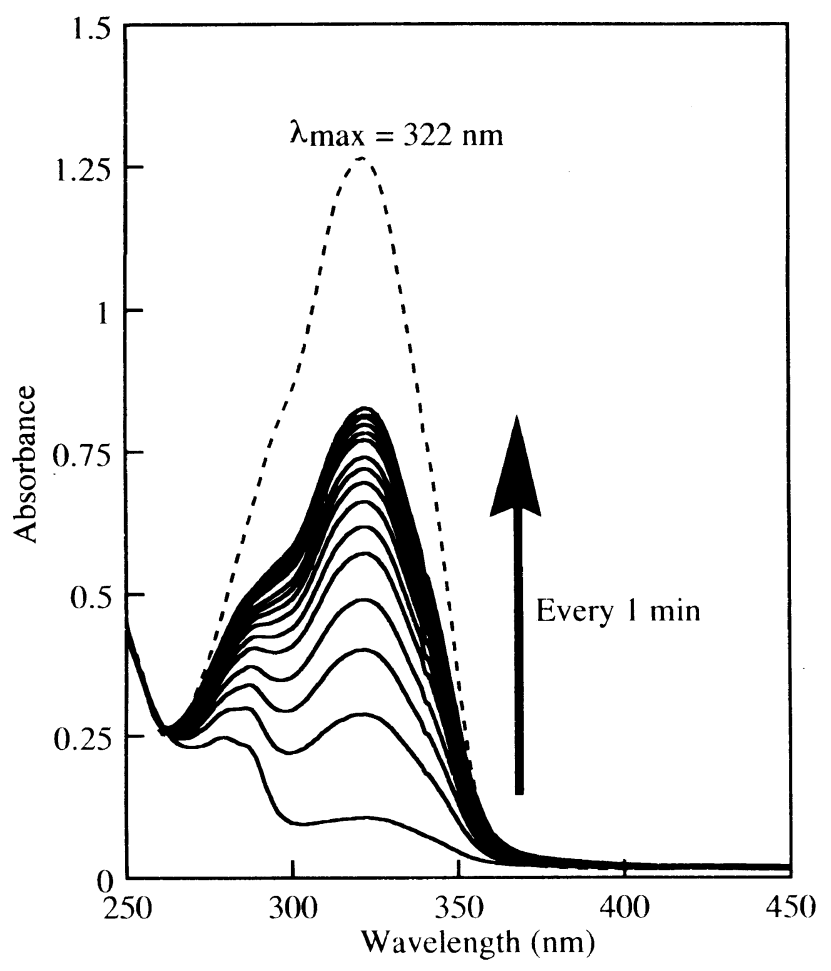

Figure 5a. The change of absorption spectra of polymer $\mathbf{4 b}$ gel during the photo-cleavage reaction induced by irradiation at $254 \mathrm{~nm}$ (every $1 \mathrm{~min})$.

methanol, but in the polymer hybrids polymer $\mathbf{4 b}$ is surrounded by rigid silica matrix and polymer must move through the pores of silica in order to be extracted. In such a fixed state, the coumarin aggregates cannot be broken by methanol and act as the physically crosslinking point. If the latter is the case, polymer $\mathbf{4 b}$ might be extracted by using DMF as a solvent, since DMF is thought to be a better solvent for both coumarin groups and polymer main chain. Actually, as shown in Figure $4 \mathrm{~b}, 73 \mathrm{wt} \%$ of the organic component was extracted by DMF before the crosslink. This indicates that polymer 4b was not covalently linked to the silica matrix, but a kind of physical crosslink might occur. After the photo-crosslinking of polymer $\mathbf{4 b}$, the extracted content was suppressed to be $c a$. $20 \mathrm{wt} \%$. Improvement of the solvent-resistant property by the formation of the IPN structure was confirmed.

In this system, both the inter- and intra-chain photodimerizations are able to occur. As a result, although the photo-dimerization was quantitative, the crosslink density should be smaller than the degree of modification $(8.2 \mathrm{~mol} \%)$. Consequently some of the polymers were extracted even after the crosslink. It was difficult, however, to determine the ratio of the inter-/intra-chain reactions.

\section{Photo-Cleavage (Reverse Photo-Dimerization) Reaction in the Polymer Hybrids}

The cyclobutane ring of the coumarin dimer is known to be cleaved by the irradiation of light shorter than $310 \mathrm{~nm} .^{8-10}$ The crosslinked polymer $4 \mathbf{b}$ film was subjected to photo-cleavage reaction by the irradiation at $254 \mathrm{~nm}$ using a low-pressure mercury lamp. Figure 5a shows the change of the absorption spectra of the

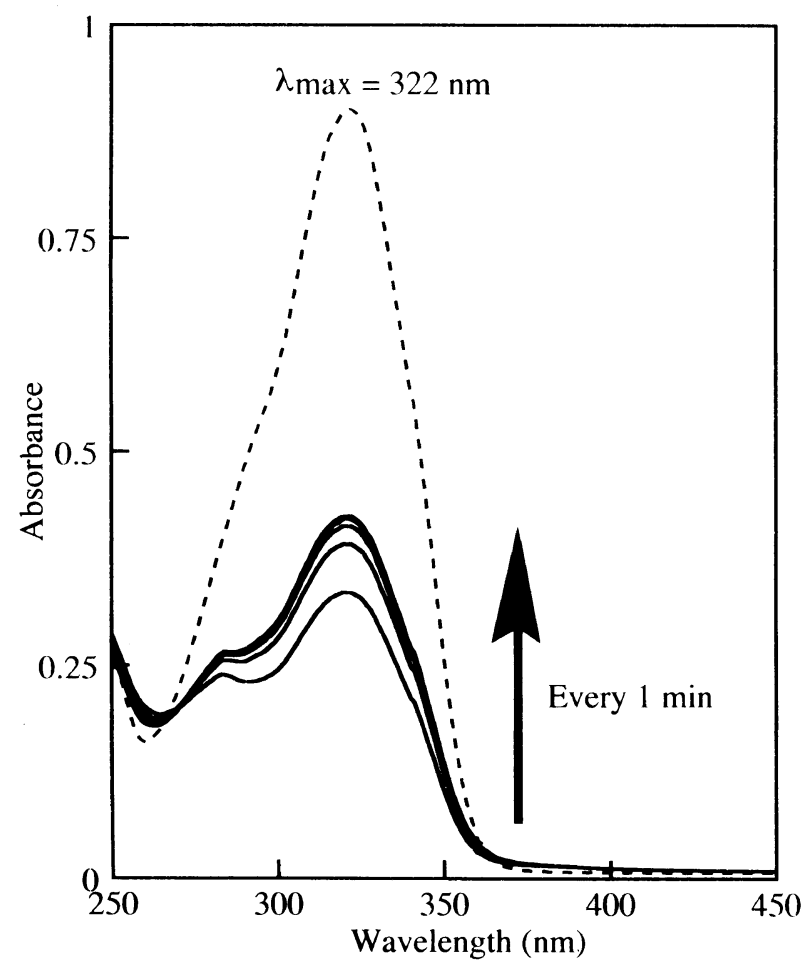

Figure 5b. The change of absorption spectra of the polymer hybrid (run 10) during the photo-cleavage reaction induced by irradiation at $254 \mathrm{~nm}$ (every $1 \mathrm{~min}$ ).

crosslinked polymer $\mathbf{4 b}$ film upon irradiation. The absorption at $322 \mathrm{~nm}$ increased gradually. This result meant that the photo-cleavage reaction did occur and the original coumarin groups were regenerated. The absorption intensity, however, did not completely recover after a long-time irradiation. Conversion of the photocleavage reaction was calculated from the absorption spectra to be $62 \%$. The reaction proceeded in all the polymer hybrid films in the same way, and about $16 \%$ of the coumarin dimer was converted to the original monomer. Figure $5 b$ shows the typical change of the absorption spectra upon irradiation on the polymer hybrid film (run 10). There are several possibilities to explain the low photo-cleavage efficiency, that is, the re-photo-dimerization by $254 \mathrm{~nm}$ light, the photo-decomposition of coumarin ring, or the formation of stable photo-dimer upon the irradiation by the high-pressure mercury lamp. ${ }^{15,16}$ However, the reason has not been clarified yet.

\section{CONCLUSIONS}

Organic-inorganic polymer hybrids were prepared from coumarin-modified polyoxazoline $\mathbf{4}$ with various coumarin contents. Transparent and homogeneous polymer hybrids were obtained with TMOS as an alkoxysilane. Acceleration of sol-gel reaction by employing 1 molar hydrochloric acid as an acid catalyst was important to obtain homogeneous polymer hybrids. Photodimerization of coumarin groups in the polymer hybrids took place almost quantitatively even with the lowest polymer ratio sample. These results were due to the tendency of the coumarin groups to aggregate in the reaction mixture. The solvent-resistant property of the 
polymer hybrids was found to be improved by the formation of IPN structure. This new strategy to prepare the IPN polymer hybrids can be applied to other polymers hanging other crosslinkable side groups. Search for more efficient crosslink and de-crosslink reactions is in progress.

\section{REFERENCES}

1. L. H. Sperling and V. Mishra, Polym. Adv. Technol., 7, 197 (1996).

2. B. M. Novak, D. Auerbach, and C. Verrier, Chem. Mater., 6, 282 (1994).

3. C. L. Jackson, B. J. Bauer, A. I. Nakatani, and J. D. Barnes, Chem. Mater., 8, 727 (1996).

4. R. Tamaki, K. Naka, and Y. Chujo, Polym. J., 30, 60 (1998).

5. R. Tamaki, K. Naka, and Y. Chujo, Polym. Bull., 39, 303 (1997).

6. N. Ramasubbu, K. Gnanaguru, K. Venkatesan, and V.
Ramamurthy, Can. J. Chem., 60, 2159 (1982).

7. N. Ramasubbu, T. N. G. Row, K. Venkatesan, V. Ramamurthy, and C. N. R. Rao, J. Chem. Soc., Chem. Commun., 178 (1982).

8. M. Hasegawa and Y. Suzuki, Chem. Lett., 317 (1972).

9. N. Yonezawa, T. Yoshida, and M. Hasegawa, J. Chem. Soc., Perkin Trans. 1, 1083 (1983).

10. Y. Chen and J.-D. Wu, J. Polym. Sci., Part A, Polym. Chem., 32, 1867 (1994)

11. Y. Chujo, K. Sada, and T. Saegusa, Macromolecules, 23, 2693 (1990).

12. C. J. Brinker and G. W. Scherer, "Sol Gel Science, The Physics and Chemistry of Sol-Gel Processing," Harcourt Brace \& Co., Publishers, Boston, MA, 1990.

13. Y. Chujo, H. Matsuki, and R. Tamaki, Polym. Prepr., Jpn., 43, E1156 (1994).

14. R. Tamaki and Y. Chujo, J. Mater. Chem., 8, 1113 (1998).

15. M. J. Moghaddam, S. Hozumi, Y. Inaki, and K. Takemoto, Polym. J., 21, 203 (1989).

16. T. Nozaki, M. Maeda, Y. Maeda, and H. Kitano, J. Chem. Soc., Perkin Trans. 2, 1217 (1997). 